\title{
AN INVESTIGATION OF SOIL PHYSICO-CHEMICAL VARIABLES ACROSS DIFFERENT LOWLAND FOREST ECOSYSTEMS OF BRUNEI DARUSSALAM
}

\author{
Salwana Md. Jaafar ${ }^{1}$, Rahayu Sukmaria Sukri ${ }^{1}$, Şerban Procheş ${ }^{2}$ \\ ${ }^{1}$ Environmental and Life Sciences Programme, Faculty of Science, Universiti Brunei Darussalam, Jalan \\ Tungku Link, BE 1410, Brunei Darussalam. \\ Email: rahayu.sukri@ubd.edu.bn \\ ${ }^{2}$ Discipline of Geography, University of KwaZulu-Natal, Westville Campus, P. B. X54001, Durban 4000, \\ South Africa \\ Received: 20 Apr 2016 Revised: 27 July 2016 Accepted: 22 Dec 2016
}

\begin{abstract}
Tropical forests undisputedly harbor the largest share of global plant diversity, but the mechanisms of maintenance for this diversity cannot be well understood without good data on environmental variables, primarily soil characteristics. This study investigates differences in soil physico-chemical properties in various tropical lowland forest types in Brunei Darussalam, in the Northwest Borneo plant diversity hotspot. Nine different vegetation types were investigated: intact primary mixed-dipterocarp forest, old disturbed secondary forest, young disturbed secondary forest (partly invaded by alien Acacias), heath (Kerangas) forest, peat swamp forest, swampy heath (Kerapah) forest, core mangrove, fringe mangrove and island mangrove forests. Nine $60 \times 20 \mathrm{~m}$ plots were set up, and sampled for soils at topsoil $(0-15 \mathrm{~cm}$ depth) and subsoil (15-30 cm depth) layers. Soil gravimetric water and organic matter content, texture, nutrient concentrations, $\mathrm{pH}$, and salinity were determined. The peat swamp and core mangrove forests recorded highest soil nutrient concentrations. Peat swamp forest had the highest GWC, $\mathrm{OM}$ content, total $\mathrm{N}$, and total $\mathrm{Ca}$ recorded, whereas the soil in core mangrove forest had higher total $\mathrm{P}$, total $\mathrm{Mg}$, total $\mathrm{K}$, exchangeable $\mathrm{Mg}$, exchangeable $\mathrm{Ca}$, exchangeable $\mathrm{K}$ and salinity compared to the other habitat types. These results were also highlighted by the principal component analysis for the soil parameters measured. The most nutrient-poor soils were found in the Kerapah and heath forest sites. The difference between topsoil and subsoil for soil variables were generally not significantly different from each other. The present study has shown that soil physico-chemical properties differ significantly between the nine vegetation types studied, and this may have important implications upon differences seen in plant community compositions in these vegetation types.
\end{abstract}

Keywords: Heath forest, Mangrove forest, Mixed Dipterocarp forest, Peat swamps, Edaphic

\section{INTRODUCTION}

Rain forests occur over a wide range of soils, with some occurring on nutrient-poor soils, others on nutrientrich soils (Proctor 1987).

These soils are diverse in terms of their physical, chemical and biological properties, thus making any generalization about rain forest soils difficult (Richards 1996). The most diverse tropical rain forest soils are typically found in the lowlands, which receive continuous rainfall affecting the nutrients input, organic matter decomposition, erosion and weathering (Ghazoul \& Sheil 2010).

This study focuses specifically on Brunei Darussalam, located in the Northwest Borneo plant diversity hotspot. The lowland Bornean forests are considered as the most diverse 
amongst the tropical rain forests in South-East Asia. The stable climatic conditions have given rise to the world's richest assemblage of plant species, as it houses high diversity of trees, approximately 10,000 species of plants, including about 267 species of Dipterocarpaceae of which 155 species are endemic to Borneo (Ashton 1989).

The underlying geology and climatic conditions of this island influence the occurrence of various soil types (Ashton 2004). The thick sedimentary rocks underlying Borneo were laid down during the Miocene and Pliocene eras (Ashton et al. 2003) and underwent rapid erosion continuously due to the wet climate. These rocks have been uplifted and eroded to create the topography which gives Brunei its distinctive landscape. The resulting weathered materials vary in composition, from sand to clay. This contributes to the great diversity of drainage and soil fertility to be found across Brunei, which results in different floras and vegetation types, characteristic to each soil type (Ashton et al. 2003, Grealish \& Fitzpatrick 2013).

The present study is the first attempt to quantify variation in soil chemical and physical properties across almost all of the lowland forest types in Brunei Darussalam. The main primary forest type in Brunei lowlands is the mixed-dipterocarp forest (Ashton et al. 2003) whereas, peat swamp forests are the second most dominant forest type (Wong \& Kamariah 1999) and followed by mangrove forests and heath (Kerangas) forest (Forestry Department 2011). Data gathered during this project will be crucial for future studies on the influence of soil properties upon the distribution of different vegetation types in Brunei, despite experiencing the same climate throughout.

The main aim of this study was to quantify and describe differences in soil physico-chemical properties across nine different vegetation types: intact primary mixed-dipterocarp forest (PMDF), old disturbed secondary forest (OSF), young disturbed secondary forest (partly invaded by alien Acacias; YSF), heath (Kerangas) forest (HF), peat swamp forest (PSF), swampy heath (Kerapah) forest $(\mathrm{KF})$, core mangrove $(\mathrm{CM})$, fringe mangrove $(\mathrm{FM})$ and island mangrove forests (IM). We analysed selected physical and chemical soil properties to determine their differences across the nine study sites, as well as differences in these soil physico-chemical properties between the topsoil (0-15 cm depth) and subsoil (15-30 cm depth) layers at each vegetation types. We also determined which soil properties explain most of the variation between the nine different vegetation types.

\section{MATERIALS AND METHODS}

The study was conducted at nine different lowland forest sites located in Brunei Darussalam, Northwest Borneo. Five of the study sites were located in Brunei-Muara District: the fringe mangrove (FM) site at Pantai Serasa, the island mangrove (IM) site at Pulau Bedukang, core mangrove (CM) site at Jalan Batu Marang, a young disturbed secondary forest (YSF) site at Jalan Kebangsaan, and old-growth secondary forest (OSF) site at Sungai Akar. The other study sites, located in the Belait 
District, were the intact primary mixed dipterocarp forest (PMDF) site at compartment 7 and heath (Kerangas) forest (HF) site at compartment 8 of the Andulau Forest Reserve in Sungai Liang, as well as the peat swamp forest
(PSF) site at Badas, and swampy heath (Kerapah) forest (KF) site at Lumut (Figure 1). Field work was carried out in March 2014 within three weeks during the season.

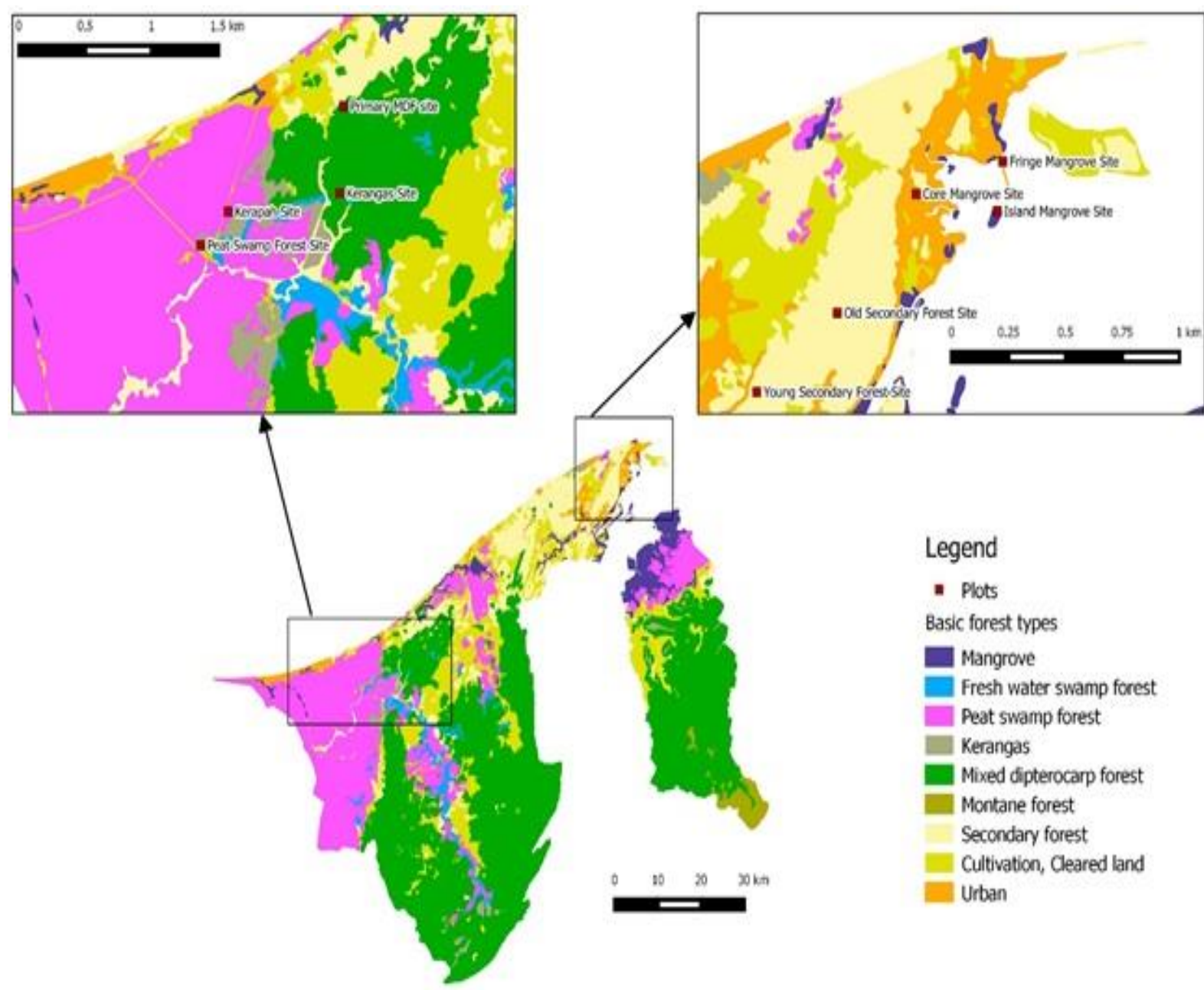

Figure 1 Map of Brunei Darussalam showing different forest types, and indicating the sites studied (Adapted from Anderson \& Marsden 1984)

\section{Soil sampling}

At each study site, a $60 \mathrm{~m} \times 20 \mathrm{~m}$ plot was randomly selected within accessible forest area, and subdivided into three $20 \mathrm{~m}$ x $20 \mathrm{~m}$ subplots, giving a total of 27 subplots for the entire study. In each $20 \mathrm{~m}$ x $20 \mathrm{~m}$ subplot, five soil 
cores were sampled using a soil auger. Soil samples were collected from the topsoil (0 - $15 \mathrm{~cm}$ depth) and subsoil (15 $-30 \mathrm{~cm}$ depth). In each subplot, four soil cores were collected close to each

\section{Soil analyses}

Gravimetric water content and $\mathrm{pH}$ in distilled water were determined using fresh soil samples (Allen et al. 1989), whereas the salinity of the soil in distilled water were determined by measuring its electrical conductivity (expressed as deci-Siemens per meter; $\mathrm{dS} / \mathrm{m})$. The electrical conductivity was measured using a YSI $63 \mathrm{pH}$, conductivity, temperature, and salinity multimeter (Rickly Hydrological Company, Columbus, Ohio, USA). The remaining samples were air-dried to constant weight, ground and sieved, and used for macronutrient analysis. Organic matter (OM) content was determined using a muffle furnace (Gallenkamp Size 2, Apeldoorn, Netherland) set at $550^{\circ} \mathrm{C}$ for two hours (Allen et al. 1989). Soil texture was determined using a modified pipette method, following the procedures of the Brunei Department of Agriculture (2006).

Total $\mathrm{N}$ and $\mathrm{P}$ concentrations were determined using the Kjeldahl method by digesting each soil sample in concentrated sulphuric acid (conc. $\mathrm{H}_{2} \mathrm{SO}_{4}$ ), and were analysed using the Flow Injector Analyser (FIAstar 5000, Hoganas, Sweden). For analysis of total $\mathrm{Mg}, \mathrm{Ca}$ and $\mathrm{K}$, air-dried soil samples were acid-digested using a microwave digestor (Multiwave 3000 Anton Paar, Austria) following the procedures of Allen et al. (1989). Exchangeable Mg, corner, and one in the center. The five samples were then mixed together as a composite bulk for each subplot. A total of 54 soil samples were collected $(n=27$ subplots $\quad \mathrm{X} \quad 2 \quad$ depths). $\mathrm{Ca}$ and $\mathrm{K}$ were extracted using $1 \mathrm{~N}$ Neutral ammonium acetate (Chapman 1965). Total and exchangeable $\mathrm{Mg}, \mathrm{Ca}$ and $\mathrm{K}$ concentrations were measured using a Flame Atomic Absorption Spectrophotometer (AAS; Thermo Scientific iCE 3300, Sydney, Australia).

Soil available $\mathrm{P}$ concentrations were extracted using Bray's solution (0.03 $\mathrm{N}$ ammonium fluoride in $0.025 \mathrm{~N}$ $\mathrm{HCl})$ and mixed with ascorbic acid and molybdate reagent (Brunei Department of Agriculture 2006). The absorbance of each solution was read at $880 \mathrm{~nm}$ wavelength using UV-spectrophotometer (UV-1800, Shimadzu, Kyoto, Japan).

\section{Data analysis}

All statistical analyses were conducted in R 3.1.1 (R Core Team, 2014). Between-site differences in soil properties were determined using oneway ANOVA and significant results further analysed using Tukey's HSD test. Variables for soil nutrient concentrations (total $\mathrm{N}, \mathrm{P}, \mathrm{Mg}, \mathrm{Ca}$ and $\mathrm{K}$, exchangeable $\mathrm{Mg}, \mathrm{Ca}$ and $\mathrm{K}$, Available P) were ln-transformed, $\mathrm{pH}$ and salinity were $\log _{10}$-transformed, and GWC and OM content were arcsinetransformed. All 13 soil variables between different sites and depth were then subjected to a principal component analysis (PCA) to determine which variables account for most of the variation in the data set. 


\section{RESULTS}

\section{Between-site differences in soil physico-chemical variables}

Soil physico-chemical variables did not differ significantly between the topsoil and subsoil depths for the sites studied, except for GWC which showed significant differences at topsoil and subsoil between sites $(\mathrm{F}=44.3, \mathrm{p}<$ 0.001 and $\mathrm{F}=55.0, \mathrm{p}<0.001$, respectively; Table 1 and Table 2). Soil GWC at PSF was consistently significantly higher than the other forest types at both sampling depths $(0-15 \mathrm{~cm}$ and $15-30 \mathrm{~cm})$, and the lowest mean GWC values were found in $\mathrm{KF}$ and $\mathrm{HF}$ soils at both sampling depths.

Soil $\mathrm{pH}$ in both the topsoil and subsoil were significantly different between sites $(\mathrm{F}=17.9, \mathrm{p}=<0.001 ; \mathrm{F}=$ $18.1, \mathrm{p}=<0.001$, respectively). Soil $\mathrm{pH}$ at two mangroves sites, FM and CM were the highest and close to neutral $\mathrm{pH}$ values, whereas $\mathrm{KF}$ and $\mathrm{HF}$ soils were the most acidic at both depths (Table 1 and Table 2). Salinity in the topsoil and subsoil were significantly different between sites $(\mathrm{F}=170.2, \mathrm{p}=<0.001 ; \mathrm{F}$ $=229.6, \mathrm{p}=<0.001$, respectively). The highest mean salinity was recorded in $\mathrm{CM}$ and the lowest was in HF soils.
OM content in the topsoil and subsoil also showed significant differences between sites $(\mathrm{F}=370.7, \mathrm{p}=<0.001 ; \mathrm{F}$ $=542.1, \mathrm{p}=<0.001$, respectively; Table 1 and Table 2). PSF soils recorded the highest mean OM content, while the lowest mean OM content were found in both IM and KF soils.

Soil texture at topsoil and subsoil depths varied between plots (see Jaafar, 2014), with some classified as sandy (FM, IM, KF and HF), while the rest were classified as loamy sand (PMDF and OSF), sandy loam (PSF and CSF) or loam (CM). Sand content ranged from $34.2 \%$ to $97.3 \%$ in all the sites studied.

At both sampling depths, CM and PSF soils were found to have the highest concentrations of nutrients, significantly higher than the other forest types. CM soils had the highest total $\mathrm{P}$, total $\mathrm{Mg}$, total $\mathrm{K}$, exchangeable $\mathrm{Mg}$, exchangeable $\mathrm{Ca}$, and exchangeable $\mathrm{K}$ concentrations recorded, whereas PSF soils had the highest total $\mathrm{N}$ and total $\mathrm{Ca}$ concentrations (Table 1 and Table 2). Total N, P, Mg, Ca, K, exchangeable $\mathrm{Mg}, \mathrm{Ca}$ and $\mathrm{K}$ and available $\mathrm{P}$ concentrations in both the topsoil and subsoil showed significant differences between sites. 
Table 1 Measurements of topsoil ( 0 - $15 \mathrm{~cm}$ depth) variables in lowland forest ecosystems of Brunei across 27 subplots from nine different sites. Soils were sampled from each 20 × 20 m subplot. Gravimetric water content (GWC) and OM content are expressed in percentage (\%). Data for GWC and OM content were arcsine-transformed prior to statistical analysis. Soil nutrient concentrations are expressed as $\mathrm{mg} \mathrm{g}^{-1}$ for total $\mathrm{N}$, total $\mathrm{Mg}$, total $\mathrm{Ca}$, total $\mathrm{K}$, exchangeable $\mathrm{Mg}$, exchangeable $\mathrm{Ca}$, exchangeable $\mathrm{K}$ and available $\mathrm{P}$ and were ln-transformed. $\mathrm{pH}$ is expressed as unit $\mathrm{pH}$ whereas salinity is expressed in $\mathrm{dS} / \mathrm{m}$. Data for $\mathrm{pH}$ and salinity were log-transformed in statistical analyses.

\begin{tabular}{|c|c|c|c|c|c|c|c|c|c|c|}
\hline $\begin{array}{l}\text { Soil } \\
\text { variables }\end{array}$ & FM & IM & $\mathbf{C M}$ & PSF & KF & HF & PMDF & OSF & YSF & \\
\hline GWC & $\begin{array}{c}34.7 \pm \\
1.1\end{array}$ & $\begin{array}{c}19.4 \pm \\
1.9\end{array}$ & $\begin{array}{c}51.9 \pm \\
7.8\end{array}$ & $\begin{array}{c}80.5 \pm \\
1.5\end{array}$ & $\begin{array}{c}17.4 \pm \\
3.1\end{array}$ & $\begin{array}{c}15.8 \pm \\
4.6\end{array}$ & $\begin{array}{c}18.0 \pm \\
0.8\end{array}$ & $\begin{array}{c}18.8 \pm \\
2.6\end{array}$ & $\begin{array}{c}17.4 \pm \\
3.1\end{array}$ & $\begin{array}{l}* \\
*\end{array}$ \\
\hline $\begin{array}{l}\text { OM } \\
\text { content }\end{array}$ & $\begin{array}{c}2.1 \pm \\
0.3\end{array}$ & $\begin{array}{l}1.9 \pm \\
0.2\end{array}$ & $\begin{array}{c}14.9 \pm \\
4.1\end{array}$ & $\begin{array}{c}85.9 \pm \\
1.4\end{array}$ & $\begin{array}{c}2.5 \pm \\
0.5\end{array}$ & $\begin{array}{c}4.2 \pm \\
1.3\end{array}$ & $\begin{array}{c}3.0 \pm \\
0.4\end{array}$ & $\begin{array}{c}3.8 \pm \\
0.3\end{array}$ & $\begin{array}{c}2.5 \pm \\
0.5\end{array}$ & * \\
\hline Total N & $\begin{array}{c}0.5 \pm \\
0.08\end{array}$ & $\begin{array}{c}0.45 \pm \\
0.07\end{array}$ & $\begin{array}{l}2.5 \pm \\
0.45\end{array}$ & $\begin{array}{c}7.8 \pm \\
2.5\end{array}$ & $\begin{array}{c}0.42 \pm \\
0.07\end{array}$ & $\begin{array}{c}0.69 \pm \\
0.15\end{array}$ & $\begin{array}{c}0.75 \pm \\
0.12\end{array}$ & $\begin{array}{c}0.9 \pm \\
0.07\end{array}$ & $\begin{array}{c}0.42 \pm \\
0.07\end{array}$ & * \\
\hline Total P & $\begin{array}{c}0.07 \pm \\
0.01\end{array}$ & $\begin{array}{l}0.05 \pm \\
0.003\end{array}$ & $\begin{array}{c}0.24 \pm \\
0.02\end{array}$ & $\begin{array}{l}0.20 \pm \\
0.03\end{array}$ & $\begin{array}{l}0.04 \pm \\
0.001\end{array}$ & $\begin{array}{l}0.03 \pm \\
0.002\end{array}$ & $\begin{array}{c}0.08 \pm \\
0.01\end{array}$ & $\begin{array}{c}0.09 \pm \\
0.01\end{array}$ & $\begin{array}{l}0.04 \pm \\
0.001\end{array}$ & * \\
\hline Total Mg & $\begin{array}{l}0.3 \pm \\
0.05\end{array}$ & $\begin{array}{l}0.15 \pm \\
0.03\end{array}$ & $\begin{array}{c}0.61 \pm \\
0.23\end{array}$ & $\begin{array}{c}0.49 \pm \\
0.04\end{array}$ & $\begin{array}{c}0.03 \pm \\
0.01\end{array}$ & $\begin{array}{l}0.03 \pm \\
0.004\end{array}$ & $\begin{array}{c}0.08 \pm \\
0.01\end{array}$ & $\begin{array}{c}0.07 \pm \\
0.004\end{array}$ & $\begin{array}{c}0.03 \pm \\
0.01\end{array}$ & $\begin{array}{l}* \\
*\end{array}$ \\
\hline Total Ca & $\begin{array}{c}0.05 \pm \\
0.01\end{array}$ & $\begin{array}{c}0.07 \pm \\
0.02\end{array}$ & $\begin{array}{c}0.03 \pm \\
0.02\end{array}$ & $\begin{array}{c}0.20 \pm \\
0.06\end{array}$ & $\begin{array}{c}0.11 \pm \\
0.02\end{array}$ & $\begin{array}{c}0.09 \pm \\
0.01\end{array}$ & $\begin{array}{c}0.01 \pm \\
0.001\end{array}$ & $\begin{array}{c}0.002 \pm \\
0.002\end{array}$ & $\begin{array}{c}0.11 \pm \\
0.02\end{array}$ & $\begin{array}{l}* \\
*\end{array}$ \\
\hline Total K & $\begin{array}{l}1.4 \pm \\
0.15\end{array}$ & $\begin{array}{c}0.73 \pm \\
0.15\end{array}$ & $\begin{array}{c}7.7 \pm \\
1.1\end{array}$ & $\begin{array}{c}0.12 \pm \\
0.02\end{array}$ & $\begin{array}{c}0.04 \pm \\
0.01\end{array}$ & $\begin{array}{c}0.01 \pm \\
0.004\end{array}$ & $\begin{array}{c}2.7 \pm \\
0.6\end{array}$ & $\begin{array}{c}3.3 \pm \\
0.9\end{array}$ & $\begin{array}{c}0.04 \pm \\
0.01\end{array}$ & $\begin{array}{l}* \\
*\end{array}$ \\
\hline $\begin{array}{l}\text { Exchange } \\
\text { able Mg }\end{array}$ & $\begin{array}{c}0.7 \pm \\
0.11\end{array}$ & $\begin{array}{c}0.34 \pm \\
0.07\end{array}$ & $\begin{array}{c}1.32 \pm \\
0.35\end{array}$ & $\begin{array}{c}0.17 \pm \\
0.01\end{array}$ & $\begin{array}{c}0.03 \pm \\
0.01\end{array}$ & $\begin{array}{c}0.03 \pm \\
0.01\end{array}$ & $\begin{array}{c}0.01 \pm \\
0.002\end{array}$ & $\begin{array}{c}0.01 \pm \\
0.001\end{array}$ & $\begin{array}{c}0.03 \pm \\
0.01\end{array}$ & $\begin{array}{l}* \\
*\end{array}$ \\
\hline $\begin{array}{l}\text { Exchange } \\
\text { able } \mathrm{Ca}\end{array}$ & $\begin{array}{c}0.35 \pm \\
0.03\end{array}$ & $\begin{array}{l}0.2 \pm \\
0.04\end{array}$ & $\begin{array}{c}0.58 \pm \\
0.18\end{array}$ & $\begin{array}{l}0.01 \pm \\
0.003\end{array}$ & $\begin{array}{l}0.01 \pm \\
0.003\end{array}$ & $\begin{array}{l}0.02 \pm \\
0.003\end{array}$ & $\begin{array}{l}0.01 \pm \\
0.001\end{array}$ & $\begin{array}{c}0.01 \pm \\
0.001\end{array}$ & $\begin{array}{l}0.01 \pm \\
0.003\end{array}$ & * \\
\hline $\begin{array}{l}\text { Exchange } \\
\text { able K }\end{array}$ & $\begin{array}{c}0.05 \pm \\
0.04\end{array}$ & $\begin{array}{l}0.1 \pm \\
0.01\end{array}$ & $\begin{array}{c}0.35 \pm \\
0.16\end{array}$ & $\begin{array}{c}0.07 \pm \\
0.01\end{array}$ & $\begin{array}{l}0.02 \pm \\
0.001\end{array}$ & $\begin{array}{l}0.04 \pm \\
0.004\end{array}$ & $\begin{array}{l}0.08 \pm \\
0.001\end{array}$ & $\begin{array}{c}0.05 \pm \\
0.01\end{array}$ & $\begin{array}{l}0.02 \pm \\
0.001\end{array}$ & $*$ \\
\hline $\begin{array}{l}\text { Available } \\
\text { P }\end{array}$ & $\begin{array}{c}0.02 \pm \\
0.001\end{array}$ & $\begin{array}{c}0.01 \pm \\
0.001\end{array}$ & $\begin{array}{c}0.01 \pm \\
0.001\end{array}$ & $\begin{array}{c}0.02 \pm \\
0.001\end{array}$ & $\begin{array}{c}0.01 \pm \\
0.001\end{array}$ & $\begin{array}{c}0.01 \pm \\
0.001\end{array}$ & $\begin{array}{c}0.01 \pm \\
0.001\end{array}$ & $\begin{array}{c}0.01 \pm \\
0.001\end{array}$ & $\begin{array}{c}0.01 \pm \\
0.001\end{array}$ & $*$ \\
\hline $\mathrm{pH}$ & $\begin{array}{l}7.2 \pm \\
0.14\end{array}$ & $\begin{array}{l}5.3 \pm \\
0.47\end{array}$ & $\begin{array}{c}5.58 \pm \\
0.82\end{array}$ & $\begin{array}{c}3.11 \pm \\
0.08\end{array}$ & $\begin{array}{c}3.1 \pm \\
0.1\end{array}$ & $\begin{array}{c}3.8 \pm \\
0.18\end{array}$ & $\begin{array}{c}4.2 \pm \\
0.09\end{array}$ & $\begin{array}{c}4.4 \pm \\
0.02\end{array}$ & $\begin{array}{c}3.1 \pm \\
0.1\end{array}$ & $\begin{array}{l}* \\
*\end{array}$ \\
\hline Salinity & $\begin{array}{l}2.1 \pm \\
0.25\end{array}$ & $\begin{array}{c}0.73 \pm \\
0.08\end{array}$ & $\begin{array}{c}3.85 \pm \\
1.67\end{array}$ & $\begin{array}{c}0.07 \pm \\
0.01\end{array}$ & $\begin{array}{c}0.01 \pm \\
0.001\end{array}$ & $\begin{array}{c}0.01 \pm \\
0.001\end{array}$ & $\begin{array}{l}0.02 \pm \\
0.003\end{array}$ & $\begin{array}{c}0.01 \pm \\
0.001\end{array}$ & $\begin{array}{c}0.01 \pm \\
0.001\end{array}$ & * \\
\hline
\end{tabular}

$* \mathrm{P} \leq 0.05, * * \mathrm{P} \leq 0.01, * * * \mathrm{P} \leq 0.001$.

FM: Fringe Mangrove , IM: Island Mangrove, CM: Core Mangrove, PSF: Peat Swamp Forest, KF: Kerapah Forest, HF: Heath Forest, PMDF: Primary Mixed-Dipterocarp Forest, OSF: Old Secondary Forest, YSF: Young Secondary Forest 
Table 2 Measurements of subsoil (15 - $30 \mathrm{~cm}$ depth) variables in lowland forest ecosystems of Brunei across 27 subplots from nine different sites. Soils were sampled from each 20 x $20 \mathrm{~m}$ subplot. Gravimetric water content (GWC) and OM content are expressed in percentage (\%).Data for GWC and OM content were arcsine-transformed prior to statistical analysis. Soil nutrient concentrations are expressed as $\mathrm{mg} \mathrm{g}^{-1}$ for total $\mathrm{N}$, total $\mathrm{Mg}$, total $\mathrm{Ca}$, total $\mathrm{K}$, exchangeable $\mathrm{Mg}$, exchangeable $\mathrm{Ca}$, exchangeable $\mathrm{K}$ and available $\mathrm{P}$ and were ln-transformed. $\mathrm{pH}$ is expressed as unit $\mathrm{pH}$ whereas salinity is expressed in $\mathrm{dS} / \mathrm{m}$. Data for $\mathrm{pH}$ and salinity were log-transformed in statistical analyses.

\begin{tabular}{|c|c|c|c|c|c|c|c|c|c|c|}
\hline $\begin{array}{l}\text { Soil } \\
\text { variables }\end{array}$ & FM & IM & CM & PSF & KF & HF & PMDF & OSF & YSF & \\
\hline GWC & $\begin{array}{c}35.5 \pm \\
0.5\end{array}$ & $\begin{array}{c}23.5 \pm \\
3.0\end{array}$ & $\begin{array}{c}50.0 \pm \\
8.9\end{array}$ & $\begin{array}{c}87.1 \pm \\
1.2\end{array}$ & $\begin{array}{c}14.9 \pm \\
1.4\end{array}$ & $\begin{array}{c}9.7 \pm \\
4.6\end{array}$ & $\begin{array}{c}16.2 \pm \\
0.6\end{array}$ & $\begin{array}{c}18.4 \pm \\
2.2\end{array}$ & $\begin{array}{c}12.9 \pm \\
0.6\end{array}$ & * \\
\hline $\begin{array}{l}\text { OM } \\
\text { content }\end{array}$ & $\begin{array}{c}2.4 \pm \\
0.2\end{array}$ & $\begin{array}{c}2.4 \pm \\
0.3\end{array}$ & $\begin{array}{c}13.2 \pm \\
3.8\end{array}$ & $\begin{array}{c}86.7 \pm \\
1.0\end{array}$ & $\begin{array}{c}1.2 \pm \\
0.2\end{array}$ & $\begin{array}{c}1.9 \pm \\
0.7\end{array}$ & $\begin{array}{c}2.1 \pm \\
0.3\end{array}$ & $\begin{array}{l}2.6 \pm \\
0.02\end{array}$ & $\begin{array}{c}2.2 \pm \\
0.1\end{array}$ & * \\
\hline Total N & $\begin{array}{l}0.5 \pm \\
0.04\end{array}$ & $\begin{array}{c}0.50 \pm \\
0.09\end{array}$ & $\begin{array}{l}2.4 \pm \\
0.50\end{array}$ & $\begin{array}{l}8.1 \pm \\
2.7\end{array}$ & $\begin{array}{c}0.17 \pm \\
0.04\end{array}$ & $\begin{array}{c}0.33 \pm \\
0.12\end{array}$ & $\begin{array}{c}0.52 \pm \\
0.08\end{array}$ & $\begin{array}{c}0.6 \pm \\
0.01\end{array}$ & $\begin{array}{c}0.48 \pm \\
0.04\end{array}$ & * \\
\hline Total P & $\begin{array}{l}0.06 \pm \\
0.002\end{array}$ & $\begin{array}{c}0.07 \pm \\
0.01\end{array}$ & $\begin{array}{c}0.23 \pm \\
0.03\end{array}$ & $\begin{array}{c}0.18 \pm \\
0.01\end{array}$ & $\begin{array}{l}0.03 \pm \\
0.004\end{array}$ & $\begin{array}{l}0.03 \pm \\
0.003\end{array}$ & $\begin{array}{c}0.08 \pm \\
0.01\end{array}$ & $\begin{array}{c}0.08 \pm \\
0.01\end{array}$ & $\begin{array}{c}0.11 \pm \\
0.01\end{array}$ & * \\
\hline Total $\mathrm{Mg}$ & $\begin{array}{l}0.4 \pm \\
0.03\end{array}$ & $\begin{array}{c}0.22 \pm \\
0.03\end{array}$ & $\begin{array}{c}0.60 \pm \\
0.18\end{array}$ & $\begin{array}{c}0.42 \pm \\
0.02\end{array}$ & $\begin{array}{c}0.04 \pm \\
0.01\end{array}$ & $\begin{array}{l}0.04 \pm \\
0.003\end{array}$ & $\begin{array}{c}0.06 \pm \\
0.02\end{array}$ & $\begin{array}{c}0.08 \pm \\
0.03\end{array}$ & $\begin{array}{c}0.17 \pm \\
0.01\end{array}$ & * \\
\hline Total Ca & $\begin{array}{c}0.06 \pm \\
0.01\end{array}$ & $\begin{array}{c}0.06 \pm \\
0.01\end{array}$ & $\begin{array}{c}0.03 \pm \\
0.01\end{array}$ & $\begin{array}{c}0.15 \pm \\
0.01\end{array}$ & $\begin{array}{l}0.13 \pm \\
0.02\end{array}$ & $\begin{array}{c}0.12 \pm \\
0.02\end{array}$ & $\begin{array}{c}0.03 \pm \\
0.02\end{array}$ & $\begin{array}{l}0.01 \pm \\
0.005\end{array}$ & $\begin{array}{c}0.003 \pm \\
0.001\end{array}$ & * \\
\hline Total K & $\begin{array}{l}1.8 \pm \\
0.17\end{array}$ & $\begin{array}{c}0.84 \pm \\
0.13\end{array}$ & $\begin{array}{c}6.7 \pm \\
0.9\end{array}$ & $\begin{array}{c}0.10 \pm \\
0.01\end{array}$ & $\begin{array}{l}0.04 \pm \\
0.004\end{array}$ & $\begin{array}{c}0.02 \pm \\
0.01\end{array}$ & $\begin{array}{c}2.7 \pm \\
0.7\end{array}$ & $\begin{array}{c}2.7 \pm \\
1.3\end{array}$ & $\begin{array}{c}4.8 \pm \\
0.7\end{array}$ & * \\
\hline $\begin{array}{l}\text { Exchangea } \\
\text { ble } \mathrm{Mg}\end{array}$ & $\begin{array}{l}0.8 \pm \\
0.05\end{array}$ & $\begin{array}{c}0.42 \pm \\
0.08\end{array}$ & $\begin{array}{c}1.66 \pm \\
0.13\end{array}$ & $\begin{array}{c}0.18 \pm \\
0.01\end{array}$ & $\begin{array}{l}0.02 \pm \\
0.002\end{array}$ & $\begin{array}{c}0.01 \pm \\
0.004\end{array}$ & $\begin{array}{c}0.01 \pm \\
0.002\end{array}$ & $\begin{array}{c}0.01 \pm \\
0.001\end{array}$ & $\begin{array}{c}0.02 \pm \\
0.004\end{array}$ & * \\
\hline $\begin{array}{l}\text { Exchangea } \\
\text { ble } \mathrm{Ca}\end{array}$ & $\begin{array}{c}0.40 \pm \\
0.01\end{array}$ & $\begin{array}{l}0.2 \pm \\
0.04\end{array}$ & $\begin{array}{c}0.57 \pm \\
0.19\end{array}$ & $\begin{array}{l}0.01 \pm \\
0.001\end{array}$ & $\begin{array}{l}0.01 \pm \\
0.001\end{array}$ & $\begin{array}{l}0.01 \pm \\
0.002\end{array}$ & $\begin{array}{c}0.01 \pm \\
0.001\end{array}$ & $\begin{array}{l}0.01 \pm \\
0.002\end{array}$ & $\begin{array}{c}0.01 \pm \\
0.002\end{array}$ & * \\
\hline $\begin{array}{l}\text { Exchangea } \\
\text { ble } \mathrm{K}\end{array}$ & $\begin{array}{l}0.08 \pm \\
0.05\end{array}$ & $\begin{array}{c}0.11 \pm \\
0.01\end{array}$ & $\begin{array}{c}0.48 \pm \\
0.21\end{array}$ & $\begin{array}{c}0.06 \pm \\
0.004\end{array}$ & $\begin{array}{l}0.01 \pm \\
0.002\end{array}$ & $\begin{array}{l}0.04 \pm \\
0.002\end{array}$ & $\begin{array}{c}0.07 \pm \\
0.003\end{array}$ & $\begin{array}{l}0.04 \pm \\
0.003\end{array}$ & $\begin{array}{c}0.07 \pm \\
0.002\end{array}$ & * \\
\hline $\begin{array}{l}\text { Available } \\
\text { P }\end{array}$ & $\begin{array}{c}0.02 \pm \\
0.001\end{array}$ & $\begin{array}{c}0.01 \pm \\
0.001\end{array}$ & $\begin{array}{c}0.01 \pm \\
0.001\end{array}$ & $\begin{array}{c}0.01 \pm \\
0.001\end{array}$ & $\begin{array}{l}0.01 \pm \\
0.001\end{array}$ & $\begin{array}{c}0.01 \pm \\
0.001\end{array}$ & $\begin{array}{c}0.01 \pm \\
0.001\end{array}$ & $\begin{array}{c}0.01 \pm \\
0.001\end{array}$ & $\begin{array}{c}0.01 \pm \\
0.001\end{array}$ & * \\
\hline $\mathrm{pH}$ & $\begin{array}{l}7.1 \pm \\
0.10\end{array}$ & $\begin{array}{c}5.3 \pm \\
0.44\end{array}$ & $\begin{array}{c}5.90 \pm \\
0.82\end{array}$ & $\begin{array}{c}3.24 \pm \\
0.03\end{array}$ & $\begin{array}{c}3.4 \pm \\
0.1\end{array}$ & $\begin{array}{c}4.1 \pm \\
0.16\end{array}$ & $\begin{array}{l}4.2 \pm \\
0.07\end{array}$ & $\begin{array}{c}4.7 \pm \\
0.04\end{array}$ & $\begin{array}{c}4.0 \pm \\
0.1\end{array}$ & * \\
\hline Salinity & $\begin{array}{c}2.2 \pm \\
0.03\end{array}$ & $\begin{array}{c}0.98 \pm \\
0.12\end{array}$ & $\begin{array}{c}3.95 \pm \\
1.68\end{array}$ & $\begin{array}{c}0.05 \pm \\
0.004\end{array}$ & $\begin{array}{c}0.01 \pm \\
0.001\end{array}$ & $\begin{array}{c}0.01 \pm \\
0.001\end{array}$ & $\begin{array}{c}0.02 \pm \\
0.001\end{array}$ & $\begin{array}{c}0.01 \pm \\
0.001\end{array}$ & $\begin{array}{c}0.04 \pm \\
0.001\end{array}$ & * \\
\hline
\end{tabular}

$* \mathrm{P} \leq 0.05, * * \mathrm{P} \leq 0.01, * * * \mathrm{P} \leq 0.001$.

FM: Fringe Mangrove , IM: Island Mangrove, CM: Core Mangrove, PSF: Peat Swamp Forest, KF: Kerapah Forest, HF: Heath Forest, PMDF: Primary Mixed-Dipterocarp Forest, OSF: Old Secondary Forest, YSF: Young Secondary Forest 


\section{Variation in soil physico-chemical properties between vegetation types}

In the principal component analysis (PCA) for the 13 topsoil parameters measured (GWC, OM content, total N, total $\mathrm{P}$, total $\mathrm{Mg}$, total $\mathrm{Ca}$, total $\mathrm{K}$, exchangeable $\mathrm{Mg}$, exchangeable $\mathrm{Ca}$, exchangeable $\mathrm{K}$, Available $\mathrm{P}, \mathrm{pH}$, and Salinity), the first three axes accounted for $86.6 \%$ of the variation in soil properties between the nine vegetation types (Table 3). The first (PC1), second (PC2), and third (PC3) axes explained
$46.3 \%, 29.3 \%$ and $11.0 \%$ of the variation seen in topsoil variables, respectively. Topsoil concentrations of total $\mathrm{Mg}$, exchangeable $\mathrm{Mg}$, exchangeable $\mathrm{Ca}$, exchangeable $\mathrm{K}$ and salinity were strongly positively associated with PC1. PC2 represented a gradient of decreasing GWC and OM content, as well as decreasing concentrations of total $\mathrm{N}$ and total $\mathrm{Ca}$ in the topsoil. PC3 represented a gradient of increasing concentrations of total $\mathrm{P}$ and total $\mathrm{K}$, but decreasing concentrations of available $\mathrm{P}$ and decreasing $\mathrm{pH}$ in the topsoil.

Table 3 Variations from principal component analysis (PCA) of one topsoil physical parameter (GWC) and twelve topsoil chemical variables (Total N, Total P, Total Mg, Total Ca, Total K, Exchangeable Mg, Exchangeable Ca, Exchangeable K, Available P, pH, Salinity and OM content) across all 27 subplots from nine different sites, and percentage of total variation explained by each principal component axis. Loadings and signs of the correlation coefficient (trait loadings) of each property for the first three principal component axes are presented. Variables with the highest loadings are indicated in bold.

\begin{tabular}{llll}
\hline Parameters & \multicolumn{3}{l}{ Principal component axes } \\
\cline { 2 - 4 } & 1 & 2 & 3 \\
\hline \% of total variation explained & 46.3 & 29.3 & 11.0 \\
Cumulative \% variation explained & 46.3 & 75.6 & 86.6 \\
& & & \\
Loadings of soil physical and chemical properties & & & \\
\hline GWC & 0.29 & $\mathbf{- 0 . 3 4}$ & -0.03 \\
OM content & 0.16 & $\mathbf{- 0 . 4 6}$ & 0.06 \\
Total N & 0.18 & $\mathbf{- 0 . 4 0}$ & 0.16 \\
Total P & 0.33 & -0.16 & $\mathbf{0 . 3 4}$ \\
Total Mg & $\mathbf{0 . 3 9}$ & -0.10 & -0.003 \\
Total Ca & 0.05 & $\mathbf{- 0 . 3 8}$ & -0.28 \\
Total K & 0.24 & 0.24 & $\mathbf{0 . 4 3}$ \\
Exchangeable Mg & $\mathbf{0 . 3 5}$ & 0.17 & -0.16 \\
Exchangeable Ca & $\mathbf{0 . 3 5}$ & 0.21 & -0.20 \\
Exchangeable K & $\mathbf{0 . 3 3}$ & 0.15 & 0.20 \\
Available P & 0.06 & -0.22 & $\mathbf{- 0 . 5 4}$ \\
pH & 0.21 & 0.30 & $\mathbf{- 0 . 4 2}$ \\
Salinity & $\mathbf{0 . 3 6}$ & 0.19 & -0.14 \\
\hline
\end{tabular}


The 27 subplots of the nine sites sampled were partitioned in ordination space and differentiated on the basis of topsoil nutrient concentrations (Figure 2). For the PSF subplots, GWC, total N, OM, available $\mathrm{P}$, and total $\mathrm{Ca}$ were the most influential set of variables. CM subplots were mostly influenced by exchangeable cation concentrations (exchangeable $\mathrm{Ca}$, $\mathrm{Mg}$ and $\mathrm{K}$ ), and also by salinity. FM subplots were influenced mostly by $\mathrm{pH}$ and total $\mathrm{K}$ concentration. The rest of the subplots (IM, KF, HF, PMDF, OSF and YSF) were not clearly distinguishable from each other based on topsoil parameters (Figure 2). The PCA for subsoil samples showed a very similar pattern with the topsoil PCA, indicating no differences in soil variation between the sampling depths (Table 4; Figure 3).

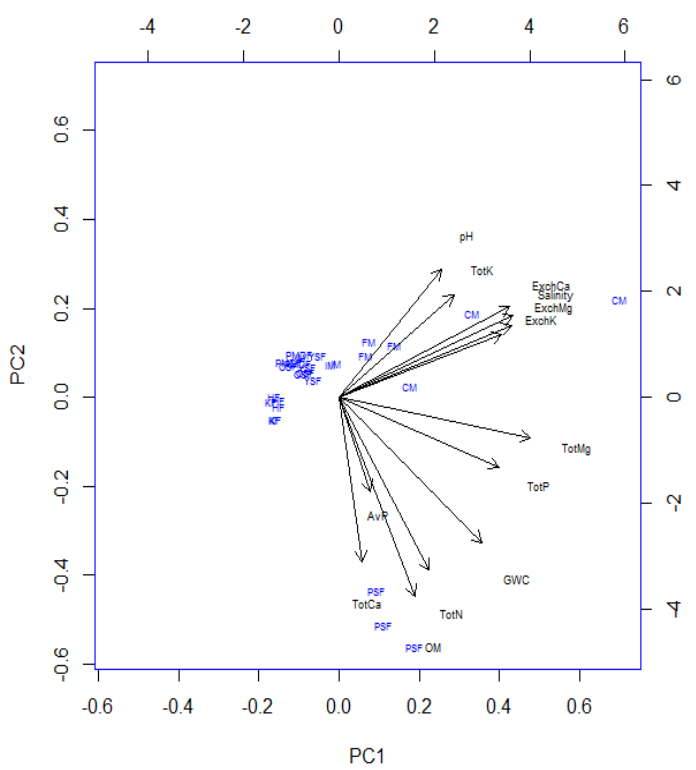

Figure 2 Biplot of principal component (PC) axes 1 and 2 from principal component analysis (PCA) of two topsoil physical properties (GWC and OM) and eleven topsoil chemical variables (Total N, Total P, Total Mg, Total Ca, Total K, Exchangeable Mg, Exchangeable Ca, Exchangeable $\mathrm{K}$, Available $\mathrm{P}, \mathrm{pH}$ and Salinity) across all 27 subplots from nine different sites.s Abbreviations denote the 27 subplots censused; FM, IM, CM, PSF, KF, HF, PMDF, OSF and YSF. GWC, OM, TotN, TotP, TotMg, TotCa, TotK, ExchMg, ExchCa, ExchK, and AvP represents gravimetric water content, organic matter content, concentrations of Total N, Total P, Total Mg, Total Ca, Total K, Exchangeable Mg, Exchangeable Ca, Exchangeable K and and Available P, respectively 


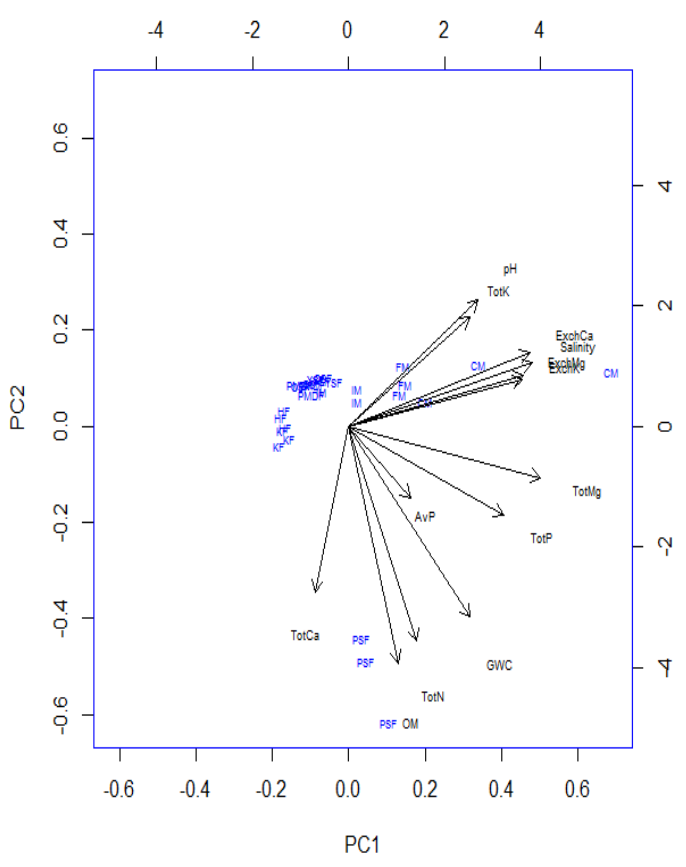

Figure 3 Biplot of principal component (PC) axes 1 and 2 from principal component analysis (PCA) of two subsoil physical properties (GWC and OM) and eleven subsoil chemical variables (Total N, Total P, Total Mg, Total $\mathrm{Ca}$, Total $\mathrm{K}$, Exchangeable Mg, Exchangeable $\mathrm{Ca}$, Exchangeable K, Available P, pH and Salinity) across all 27 subplots from nine different sites. Abbreviations denote the 27 subplots censused; GWC, OM, TotN, TotP, TotMg, TotCa, TotK, ExchMg, ExchCa, ExchK, and AvP represents gravimetric water content, organic matter content, concentrations of Total N, Total P, Total Mg, Total Ca, Total K, Exchangeable Mg, Exchangeable $\mathrm{Ca}$, Exchangeable $\mathrm{K}$ and and Available P, respectively 
Table 4 Variations from principal component analysis (PCA) of one subsoil physical parameter (GWC) and twelve subsoil chemical variables (Total N, Total P, Total Mg, Total Ca, Total K, Exchangeable Mg, Exchangeable Ca, Exchangeable K, Available P, pH, Salinity and OM content) across all 27 subplots from nine different sites and percentage of total variation explained by each principal component axis. Loadings and signs of the correlation coefficient (trait loadings) of each property for the first three principal component axes are presented. Variables with the highest loadings are indicated in bold.

\begin{tabular}{|c|c|c|c|}
\hline \multirow[t]{2}{*}{ Parameters } & \multicolumn{3}{|c|}{ Principal component axes } \\
\hline & 1 & 2 & 3 \\
\hline$\%$ of total variation explained & 47.6 & 27.4 & 12.1 \\
\hline Cumulative $\%$ variation explained & 47.6 & 75.0 & 87.1 \\
\hline \multicolumn{4}{|c|}{$\begin{array}{l}\text { Loadings of soil physical and chemical } \\
\text { properties }\end{array}$} \\
\hline GWC & 0.24 & -0.40 & -0.009 \\
\hline OM content & 0.10 & -0.50 & -0.10 \\
\hline Total N & 0.14 & -0.45 & -0.12 \\
\hline Total P & 0.31 & -0.19 & -0.39 \\
\hline Total Mg & 0.39 & -0.11 & 0.05 \\
\hline Total Ca & -0.07 & -0.35 & 0.37 \\
\hline Total K & 0.24 & 0.23 & -0.44 \\
\hline Exchangeable $\mathrm{Mg}$ & 0.35 & 0.11 & 0.08 \\
\hline Exchangeable $\mathrm{Ca}$ & 0.37 & 0.16 & 0.21 \\
\hline Exchangeable K & 0.35 & 0.10 & -0.13 \\
\hline Available P & 0.12 & -0.15 & 0.53 \\
\hline $\mathrm{pH}$ & 0.26 & 0.27 & 0.35 \\
\hline Salinity & 0.37 & 0.14 & 0.12 \\
\hline
\end{tabular}

\section{DISCUSSION}

\section{Soil variation in Brunei's lowland forests}

Our study has revealed considerable variation in soil physical and chemical properties between the nine different lowland vegetation types in Brunei. Overall, all soils sampled were acidic, except for the fringe mangrove (FM) and core mangrove (CM) soils which were close to neutral $\mathrm{pH}$ values.
Soils generally showed low OM content, except for PSF soils which showed very high values exceeding $80 \%$. Soil nutrient concentrations also varied between vegetation types.

Some of the most remarkable results obtained here were relevant to peat swamp forests. The high OM content in PSF soils recorded indicates OM production exceeded its decomposition rate (Page et al. 2006, Whitten et al. 2000). The acidic conditions of PSF soils 
also slow down the decomposition rates further (Baillie 1996, Tie 1990, Whitten et al. 2000). In addition, PSF had significantly higher mean GWC than the other forest types, and this is also visible in the PCA biplots (Figure 2 and Figure $3)$. The high water content in PSF soil is derived mainly from precipitation (Rieley et al. 1996, Yule 2010), which could also be the cause of high OM content on its peat layer. The very slow decomposition in PSF seems to immobilize nutrients, and these nutrients can reside in OM for many years rather than being released into soil (Baillie 1996, Tie 1990). The accumulation of $\mathrm{OM}$ directly affects the retention of water in the soil, and thus increases GWC. The availability of nutrients for plants is also dependent on soil GWC (Morris et al. 2011). High soil GWC typically increase nutrient availability in the soil, because nutrients become labile in the presence of water and are more easily absorbed by plant roots (Hager et al. 1991, Yule \& Gomez 2009).

The highest concentrations of total $\mathrm{N}$ and $\mathrm{Ca}$ were also recorded in the PSF plots. The PCA biplots (Figure 2 and Figure 3) clearly show a separation of the PSF subplots from the other subplots due to a high influence of total $\mathrm{N}$ and $\mathrm{Ca}$, and to a lesser extent, available $P$ concentrations. This may be partly due to sampling of peat, rather than mineral soil, at the PSF site. Soil sampling procedures in this study were standardized at $0-15$ $\mathrm{cm}$ for topsoil and $15-30 \mathrm{~cm}$ for subsoil samples. As peat depth can range from $0.5 \mathrm{~m}$ to in excess of $10 \mathrm{~m}$ (Anderson et al. 1983, Hooijer 2006, Whitten et al. 2000, Yule \& Gomez 2009, Yule 2010), it is possible that soil samples from the
PSF site were mainly comprised of peat. This is not necessarily a drawback though, as most of the PSF plants directly access nutrients from the thick OM accumulating in the peat rather than directly from the soil (Jackson et al. 2008, Page et al. 2006, Rieley et al. 1996).

In the PCA, all the three mangrove sites were found to be different from each other. The island mangrove (IM) was indistinguishable from the rest of the forest types, except for the CM, FM and PSF. Core mangrove (CM) plots had the highest mean of total $\mathrm{P}, \mathrm{Mg}, \mathrm{K}$, exchangeable $\mathrm{Mg}, \mathrm{Ca}$ and $\mathrm{K}$ concentrations recorded. This may be due to its location near the urban areas in Jalan Batu Marang, very closely located to the nearby river, Sungai Batu Marang. Nutrient availability may be increased due to the continuous flushing down of nutrients from neighbouring rivers and streams (Hogarth 1996, Morgany et al. 1999).

Another possible reason, not involving anthropogenic effects, could be that the permanently waterlogged condition at the $\mathrm{CM}$ site causes accumulation of $\mathrm{OM}$ made up of underground portions of mangrove root systems on the soil (Arianto et al. 2015, Tomlinson 1986), creating higher cation concentrations as the nutrients can also be derived directly from the accumulation of OM (Thomas 1970, Clark et al. 2001). In this way, CM ecosystem could be considered as a nearly closed system, which only continuously receives nutrient input via OM accumulation and decomposition, but has little or no nutrient leakage, thus retains a high 
concentration of nutrients (Whitmore 1984).

One of the mangrove sites studied, the FM, could be seen clearly in the PCA biplot as being highly influenced by $\mathrm{pH}$ and total $\mathrm{K}$ concentrations. Due to its high concentration of total $\mathrm{K}$ ions, the $\mathrm{pH}$ was also recorded as the highest, close to neutral. The $\mathrm{K}$ ions were likely mainly supplied by the neighbouring seawater residing in the soil of the FM. We also recorded low $\mathrm{pH}$ in all of our sites, except for the mangrove sites. Grealish and Fitzpatrick (2013) determined that acid sulphate soils in Brunei Darussalam are generally of low $\mathrm{pH}$ and strongly suggested that sulphur is present in the soils of Brunei's forests. Thus, most forest soils in Brunei are acid sulphate soils with low $\mathrm{pH}$, as detected in our study. In contrast, $\mathrm{CM}$ soils are not acid sulphate soils (Kathiresan \& Bingham 2001) and so their $\mathrm{pH}$ values tend to be higher.

The most nutrient-limited soils appeared to occur at the $\mathrm{HF}, \mathrm{KF}$ and to a lesser extent, PMDF sites. Although low levels of $\mathrm{N}$ were recorded previously from HF soil solutions (Moran et al. 2000), higher levels of $\mathrm{N}$ in soils have been recorded in $\mathrm{HF}$ at Bukit Sawat, Brunei (Metali et al. 2015). In our study, the low levels of total $\mathrm{N}$ in the HF and KF soils was probably due to the rapid uptake of $\mathrm{N}$ into the plant biomass in order to sustain the production of sclerophyllous leaves that are typical of $\mathrm{KF}$ and $\mathrm{HF}$ vegetation (Turner et al. 2000). The plant communities of KF and HF are closer in composition compared to PMDF (Forestry Department 2011), and so it is possible that they utilize similar nutrients, thus both may have similar requirements for $\mathrm{N}$. The low $\mathrm{pH}$ recorded on both HF and KF soils increased their acidity and therefore caused lower total and available P. Nutrient retention tend to be low in heath forests (Becker \& Wong 1993) due to intense leaching and the soils' inability to retain cations (Proctor 1999), resulting in soil acidification due to increased hydrogen ions (Metali et al. 2015).

Patterns in available phosphorus (P) concentrations in the soils sampled here were particularly interesting. High available $\mathrm{P}$ concentrations were recorded in all sites except for PMDF, which recorded the lowest available $P$ concentrations at both depths. Tropical forests are most often limited by $\mathrm{P}$ (Sollins 1998) and $\mathrm{P}$ has been shown to be an important nutrient for Bornean tropical soils (Paoli et al. 2006, Sukri et al, 2012). The low available $\mathrm{P}$ concentration detected here may mean that the plant roots in the PMDF site take up available $P$ in the soils effectively because available $\mathrm{P}$ is the form that is more accessible to plants than total $\mathrm{P}$ (Sollins 1998). The low available $P$ concentrations in the soil possibly indicates that it is a nutrient that is in high demand by plants and therefore it is absorbed very quickly by the plants in PMDF. Available $\mathrm{P}$ concentrations in Andulau and another PMDF site in Brunei, Belalong, were also recorded as very low (Metali et al. 2015, Sukri et al. 2012).

Kerapah forest (KF) was the forest type that was least investigated prior to our study. We found that both KF and HF have significantly low total $\mathrm{N}, \mathrm{P}, \mathrm{Mg}, \mathrm{K}$, exchangeable $\mathrm{Mg}, \mathrm{Ca}$, and $\mathrm{K}$ 
concentrations for both depths, as well as low GWC values. The low GWC in KF and HF soils could mean that there is very little water retention in these soils due to their porous and sandy nature (Brunig 1974, Katagiri et al., 1991, Proctor et al. 1983, Proctor 1999, Whitmore 1984), and therefore this could be an indication of nutrient leaching (Davies \& Becker 1996, Moran et al. 2000). HF by nature is located on sandy soils (Whitmore 1984) and, in general, sandy soils do not contain a lot of nutrients (Proctor et al. 1983). The amount of nutrients and salts are dependent upon the amount of water present in the soil (Biswas \& Mukherjee 1994). Thus, sandy soils with low water content tend to lose nutrients more easily than other soil types. As only three plots were each sampled in $\mathrm{HF}$ and $\mathrm{KF}$, the results from this study should not be generalized to all $\mathrm{HF}$ and $\mathrm{KF}$ in Brunei and in Borneo. Nevertheless, the low water availability in both HF (Richards 1996, Whitmore 1984) and KF soils are known to limit the decomposition rates, thus reducing nutrient release into the soil (Proctor 1999, Villela \& Proctor 2002).

A comparison of this current study and other soil studies based in Brunei's forests in terms of differences in nutrient concentrations is limited due to differences in methodologies used (Moran et al. 2000, Sukri et al. 2012) or because most previous studies in Brunei did not measure all soil nutrient concentrations, $\mathrm{pH}$, salinity or soil physical properties investigated in this study (Ashton \& Hall 1992, Austin et al. 1972). In addition, most of these previous studies were conducted in PMDF (Ashton \& Hall 1992, Austin et al. 1972, Moran et al. 2000, Sukri et al. 2012). Moran et al.
(2000) measured soil nutrient concentrations in a 1 ha permanent plot at Andulau, but measurements were made from soil solution rather than from the bulk soil, and so are not directly comparable to the results obtained in this present study. Nevertheless, Moran et al. (2000) found similar patterns of low $\mathrm{N}$ concentrations in their $\mathrm{HF}$ site at Badas Forest Reserve (4'34', 114 ${ }^{\circ} 25^{\prime} \mathrm{E}$; 11-16 m elevation a. s. 1.). Additionally, the data recorded for this study are comparable with the data from Matali \& Metali (2015) for HF soils, conducted at the same locality in compartment 8 of the Andulau Forest Reserve and with the data from Metali et al. (2015) for MDF soils, conducted at the same compartment 7 of the Andulau Forest Reserve in Sungai Liang.

Regarding the dearth of soil depth differences, subsoil samples in our study were only taken at $15-30 \mathrm{~cm}$ depth, and were not as deep as in other studies (see Sukri et al. 2012); thus, most nutrient concentrations were not significantly different between topsoil and subsoil. It is likely that increasing sampling depth to $30-50 \mathrm{~cm}$ for subsoil samples similar to that of Sukri et al. (2012) would result in significant differences in soil properties between depths. Nevertheless, most nutrient uptake occur at the topsoil where most of the plant roots are concentrated (Jobbagy \& Jackson 2001) and where the biological recycling of cations is most active (Baillie 1996), or nutrients may be supplied directly from the weathering of parent rocks (Proctor 1987). Therefore, an attempt was made here to determine whether differences could be noted between closer soil layers. The general failure to detect one suggests that, for 
most purposes, future studies can simply focus on topsoil properties.

\section{CONCLUSION}

The present study has explored a number of forest types for which information was previously lacking, in particular swampy heath (Kerapah) forest, which was found to be largely similar to heath (Kerangas) forest in terms of the physico-chemical properties of the soil. The inclusion of mangrove forest types here is also viewed as an innovative contribution, as these are often treated as a marine system, and comparison with fully terrestrial forest types are limited. Indeed, insofar as the adaptations of mangrove trees belonging to the same lineages as fully terrestrial ones are to be understood, such standardized approaches are needed. The analyses conducted here confirm the distinctness of these ecosystems, but also highlight major differences between mangrove forest sites at different locations.

The data set assembled here is one of the most comprehensive data sets covering a variety of tropical forest types, and thus provides an opportunity for understanding the factors filtering major plant lineages across soil types in a climatically homogeneous environment. At the same time, the breadth of the system types covered here had to be compensated by limited sampling in each type, and so the thorough characterization of those forest types first analysed here will require further replication.

\section{ACKNOWLEDGEMENTS}

We thank the Brunei Forestry Department for granting permission to conduct research at the nine sites studied (permit no. [36]/JPH/UND/17 Pt.1), and Universiti Brunei Darussalam for research support. We also thank technical staff of Environmental and Life Sciences Programme for laboratory assistance, Nurqayah, Hakeem, Hafizah, Zulfadliudin, Khalish, Norafizah, Hazirah and Yasmin for field assistance and Dr. Faizah Haji Metali for advice on soil nutrient analysis. We thank Rodzay Abd Wahab for assistance with the map.

\section{REFERENCES}

Allen S. E., Grimshaw H. M., Parkinson J. A. and Quarmby C. (1989). Chemical analysis of ecological materials. Blackwell, Oxford.

Anderson J. M., Proctor J. and Vallack H. W. (1983). Ecological studies in four contrasting lowland rain forests in Gunung Mulu National Park, Sarawak. III. Decomposition processes and nutrient losses from leaf litter. Journal of Ecology 71: 503-528.

Anderson J. A. R. and Marsden, J. D. (1984). The Forest Resources of Brunei Darussalam. Brunei Forest Resources and Strategy Planning Study 1

Arianto C. I., Gandaseca S., Rosli N., Pazi A. M. M., Ahmed O. H., Hamid H. A. and Majid, N. M. A. (2015). Soil carbon storage in dominant species of mangrove forest of Sarawak, Malaysia. International Journal of Physical Sciences 10: 210-214. 
Ashton P. S. (1989). Species richness in tropical forests. In: Tropical forests (eds. Hohn-Nielsen L. B., Nielsen I. C. and Biaslev H.). Academic Press, London, pp. 239-251.

Ashton P. S. and Hall P. (1992). Comparisons of structure among dipterocarp forests of northwestern Borneo. Journal of Ecology 80: 459481.

Ashton P. S., Kamariah A. S. and Idris M. S. (2003). A field guide to the forest and trees of Brunei Darussalam and the northeast Borneo hotspot, Vol. 1. Universiti Brunei Darussalam, Bandar Seri Begawan, Brunei Darussalam.

Ashton P. S. (2004). Soils in the tropics. In: Tropical forest diversity and dynamism: findings from a large-scale plot network (eds. Losos E. C. and Leigh E. G.) University of Chicago Press, Chicago, pp. 90-102.

Austin M. P., Ashton P. S. and GreigSmith P. (1972). The application of quantitative methods to vegetation survey. III. A re-examination of rain forest data from Brunei. Journal of Ecology 60: 305-324.

Baillie I. C. (1996). Soils of the humid tropics. In: The tropical rain forest, $2^{\text {nd }}$ edition (eds. Richards P. W.) Cambridge University Press, Cambridge, pp. 256-286.

Becker P. and Wong M. (1993). Droughtinduced mortality in tropical heath forest. Journal of Tropical Forest Science 5: 416-419.

Biswas T. D. and Mukherjee S. K. (1994). Textbook of soil science, $2^{\text {nd }}$ edition. Tata McGraw-Hill Publishing Company Limited, New Delhi, India.

Brunig E. F. (1974). Ecological studies in the Kerangas forests of Sarawak and Brunei. Borneo Literature Bureau for
Sarawak Forest Department, Kuching, Sarawak.

Chapman H. D. (1965). Cation-exchange capacity. Methods of soil analysisChemical and microbiological properties. Agronomy 9: 891-901.

Clark D. A., Brown S., Kicklighter D.W., Chambers J. Q., Thomlinson J. R., Ni J. and Holland E. A. (2001). Net primary production in tropical forests: an evaluation and synthesis of existing field data. Ecological Applications 11: 371-384.

Davies S. J. and Becker P. (1996). Floristic composition and stand structure of mixed dipterocarp and heath forests in Brunei Darussalam. Journal of Tropical Forest Science 8: 542-569.

Department of Agriculture. (2006). Laboratory Manual for Soil and Plant Analysis. Ministry of Industry and Primary Resources, Brunei. Soil and Plant Nutrition Unit, Agrotechnology Development Division.

Forestry Department. (2011). Sustainable forestry in Brunei Darussalam. Ministry of Industry and Primary Resources, BSB, Brunei.

Ghazoul J. and Sheil D. (2010). Tropical rain forest ecology, diversity and conservation. Oxford University Press, United Kingdom.

Grealish G. J. and Fitzpatrick R. W. (2013). Acid sulphate soil characterization in Negara Brunei Darussalam: a case study to inform management decisions. Soil Use and Management 29: 432-444.

Hager J. P., Warren G. P., Beer J. W. and Kass D. (1991). Phosphorus availability under alley cropping and mulched and un-mulched sole cropping systems in 
Costa Rica. Plant and Soil 137: 275283.

Hogarth P. J. (1996). The Biology of Mangroves. Oxford University Press, New York.

Hooijer A. (2006). Tropical peatlands in Southeast Asia. In: The biology of peatlands (eds. Rydin H. and Jeglum J. K.) Oxford University Press, Oxford.

Jaafar S. M. (2014). An investigation of soil physico-chemical variables across different lowland forest ecosystems of Brunei Darussalam. BSc. Thesis, Universiti Brunei Darussalam, Bandar Seri Begawan, Brunei Darussalam.

Jackson C. R., Liew K. C. and Yule C. M. (2008). Structural and functional changes with depth in microbial communities in tropical peat swamp forest sediments. Microbial Ecology 57: 402-412.

Jobbagy E. G. and Jackson R. B. (2001). The distribution of soil nutrients with depth: Global patterns and the imprint of plants. Biogeochemistry 53: 51-77.

Katagiri S., Yamakura Y. and Lee S. H. (1991). Properties of soils in kerangas forest on sandstone at Bako National Park, Sarawak, East Malaysia. Southeast Asian Studies 29, 35-48.

Kathiresan K. and Bingham L. (2001). Biology of mangroves and mangrove ecosystems. Advances in Marine Biology 40: 82-251.

Matali S. and Metali F. (2015). Selected soil physico-chemical properties in the Acacia mangium plantation and the adjacent heath forest at Andulau Forest Reserve. Malaysian Journal of Soil Science 19: 45-48.

Metali F., Kamariah A. S., Tennakoon K. and Burslem D. F. R. P. (2015). Controls on foliar nutrient and aluminum concentrations in a tropical tree flora: phylogeny, soil chemistry and interactions among elements. New Phytologist 205: 280-292.

Moran J. A., Barker M. G., Moran A. J., Becker P. and Ross S. M. (2000). A comparison of the soil water, nutrient status, and litterfall characteristics of Tropical Heath and Mixed-Dipterocarp Forest sites in Brunei. The Journal of Tropical Biology and Conservation 32: 2-13.

Morgany T., Sivasothi N., Murphy D. H., Ng P. K. L., Soong B. C., Tan H. T. W. and Tan T. K. (1999). The ecosystem and plant diversity. Title ?? In: A guide to the mangroves of Singapore 1 (eds. Ng P. K. L. and Sivasothi N.) Omnitheatre Singapore Science Centre, Singapore., pp, 15-16.

Morris T. L., Esler K.J., Barger N. N., Jacobs S. M. and Creamer M. D. (2011). Ecophysiological traits associated with the competitive ability of invasive Australian Acacias. Diversity and Distribution 17: 898-910.

Page S. E., Rieley J. O. and Wust R. (2006). Lowland tropical peatlands of Southeast Asia. In: Peatlands: Evolution and Records of Environmental and Climate Changes (eds. Martini I. P., Martinez C. A., Chesworth W.) Elsevier, Amsterdam, pp, 145-172.

Paoli G. D., Curran L. M. and Zak D. R. (2006). Soil nutrients and beta diversity in the Bornean Dipterocarpaceae: Evidence for niche partitioning by tropical rain forest trees. Journal of Ecology, 94: 157-170.

Proctor J., Anderson J. M., Chai P. and Vallack H. W. (1983). Ecological studies in four contrasting lowland rain forests in Gunung Mulu National Park, 
Sarawak. I. Forest environment, structure and floristics. Journal of Ecology 71: 237-260.

Proctor J. (1987). Nutrient cycling in primary and old secondary rainforests. Applied Geography 7: 135-152.

Proctor J. (1999). Heath forests and acid soils. Botanical Journal of Scotland 51: 1-14.

R Development Core Team. (2012). R: A language and environment for statistical computing. R Foundation for Statistical Computing, Vienna, Austria. ISBN 3-900051-07-0, URL http://www.R-project.org/.

Richards P. W. (1996). The tropical rain forest, $2^{\text {nd }}$ edition. Cambridge University Press, Cambridge.

Rieley J. O., Ahmad-Shah A. A. and Brady M. A. (1996). The extent and nature of tropical peat swamps. In: Tropical lowland peatlands of Southeast Asia (eds. Maltby E., Immirzi C. P. and Safford R. J.) IUCN, Switzerland, pp. 17-53.

Sollins P. (1998). Factors influencing species composition in tropical lowland rain forest: Does soil matter? Ecology 79: 23-30.

Sukri R. S., Wahab R. A., Salim K. A. and Burslem D. F. R. P. (2012). Habitat associations and community structure of Dipterocarps in response to environment and soil conditions in Brunei Darussalam, Northwest Borneo. Biotropica 44: 595-605.

Tie Y. L. (1990). Studies of peat swamps in Sarawak with particular reference to soil-forest relationships and development of dome-shaped structures. Dissertation, Polytechnic of
North London, London, United Kingdom.

Thomas G. W. (1970). Soil and climate factors which affect nutrient mobility. In: Nutrient mobility in soils: accumulation and losses (eds. Engelstad O. P.) Soil Science Society of America, Inc., Wisconsin, USA.

Tomlinson P. B. (1986). The Botany of Mangroves. Cambridge University Press, Cambridge.

Turner I. M., Lucas P. W., Becker P., Wong S. C., Yong W. H., Choong M. F. and Tyree M. T. (2000). Tree leaf form in Brunei: a heath forest and a mixed dipterocarp forest compared. Biotropica 32: 53-61.

Villela D. M. and Proctor J. (2002). Leaf litter decomposition and monodominance in the Peltogyne forest of Maracá Island, Brazil. Biotropica 34: 334-347

Whitmore T. C. (1984). Tropical rain forests of the Far East. Second Edition. Oxford Science Publications: Oxford.

Whitten T., Soeriaatmadja R. E. and Afiff S. A. (1997). The ecology of Java and Bali. Oxford University Press, Oxford.

Wong K. M. and Kamariah A. S. (1999). Forests and trees of Brunei Darussalam. Universiti Brunei Darussalam, Bandar Seri Begawan, Brunei Darussalam.

Yule C. M. and Gomez L. N. (2009). Leaf litter decomposition in a tropical peat swamp forest in Peninsular Malaysia. Wetlands Ecology and Management, 17: 231-241.

Yule C. M. (2010). Loss of biodiversity and ecosystem functioning in IndoMalayan peat swamp forests. Biodiversity Conservation, 19: 393-409. 